University of Nebraska - Lincoln

DigitalCommons@University of Nebraska - Lincoln

4-15-1997

\title{
Metamagnetic domains and dynamic fluctuations in $\mathrm{FeBr}_{2}$
}

O. Petracic

Laboratorium fu"r Angewandte Physik, Gerhard-Mercator-Universita"t Duisburg, D-47048 Duisburg,

Christian Binek

University of Nebraska-Lincoln, cbinek@unl.edu

Wolfgang Kleeman

Laboratorium fu"r Angewandte Physik, Gerhard-Mercator-Universita"t Duisburg, D-47048 Duisburg, wolfgang.kleemann@uni-due.de

Follow this and additional works at: https://digitalcommons.unl.edu/physicsbinek

Part of the Physics Commons

Petracic, 0.; Binek, Christian; and Kleeman, Wolfgang, "Metamagnetic domains and dynamic fluctuations in $\mathrm{FeBr}_{2}$ " (1997). Christian Binek Publications. 9.

https://digitalcommons.unl.edu/physicsbinek/9

This Article is brought to you for free and open access by the Research Papers in Physics and Astronomy at DigitalCommons@University of Nebraska - Lincoln. It has been accepted for inclusion in Christian Binek Publications by an authorized administrator of DigitalCommons@University of Nebraska - Lincoln. 


\title{
Metamagnetic domains and dynamic fluctuations in $\mathrm{FeBr}_{2}$
}

\author{
O. Petracic, Ch. Binek, and W. Kleemann \\ Laboratorium für Angewandte Physik, Gerhard-Mercator-Universität Duisburg, D-47048 Duisburg, \\ Germany
}

The mixed phase and the regime of non-critical fluctuations of the magnetic phase diagram of $\mathrm{FeBr}_{2}$ is investigated by SQUID susceptometry and light diffraction techniques. The experiments seem to evidence instability of the tricritical point as conjectured recently. The observation of stripe domains and light diffraction below and above $T_{m}=4.6 \mathrm{~K}$ and the virtual continuation of the phase transition line to above $T_{m}$ are in agreement with the occurrence of a critical endpoint at $T_{m}$ and of a bicritical endpoint at $T>T_{m}$. (C) 1997 American Institute of Physics. [S0021-8979(97)15308-7]

The magnetic $H-T$ phase diagram (PD) of the layered hexagonal antiferromagnet $\mathrm{FeBr}_{2}$ (space group $D_{3 d}^{3}$ ), where $H$ and $T$ are the applied axial magnetic field and the temperature $T$, respectively, has recently attracted considerable renewed interest. ${ }^{1}$ Obviously the topology of its multicritical point (MCP: $T_{m}=4.64 \mathrm{~K}, H_{m}=2.45 \mathrm{MA} / \mathrm{m}$ ) is more complex than that of the classical tricritical point (TCP), of the related rhombohedral compound $\mathrm{FeCl}_{2}$ (space group $D_{3 d}^{5}$ ). On the one hand, its second- and first-order phase transition (PT) lines, $H_{c}(T)$ and $H_{c 1}(T)$ [Fig. 1, straight lines obtained from peaks of $d M(H) / d H$ and $d M(T) / d T$, respectively], meet under an oblique angle at $T_{m}$ (arrow). Owing to demagnetization effects the horizontal first-order PT line splits up into a mixed phase regime within $H_{c 1}=2.33 \mathrm{MA} / \mathrm{m}<H<H_{c 2}$ $=2.75 \mathrm{MA} / \mathrm{m}$. On the other hand, strong noncritical fluctuations $(\mathrm{NCF})^{1}$ give rise to pronounced peaks of the out-ofphase components, $\chi^{\prime \prime}(H)$, of the axial ac susceptibility at low-frequencies above $T_{m}$ at $H_{-}(T)$ and $H_{+}(T)$, respectively (Fig. 1, dashed lines).

Originally ${ }^{1}$ the NCF were attributed to intraplanar frustrated exchange being ferromagnetic (FM) and antiferromagnetic (AF) between nearest and third-nearest neighbor spins of the $\mathrm{Fe}^{2+}$ ions, respectively. Indeed, Monte Carlo-type model calculations on a two-dimensional triaxial ANNNI model show characteristic features of slow NCF at $H<H_{c} .{ }^{1}$ Based on the same model Hamiltonian extended by weak interlayer $\mathrm{AF}$ exchange, Selke et al. ${ }^{2}$ and ArugaKatori et $\mathrm{al}^{3}$ recently carried out Monte Carlo and cluster variation calculations, which yield extra shoulders and peaks, respectively, of the magnetic specific heat $c_{m}$ below $T_{c}(H)$. In particular, Selke ${ }^{2}$ pointed out that the extraordinarily large coordination number, $N=20$, of nearest interplanar $\mathrm{AF}$ bonds in $\mathrm{FeBr}_{2}$ is essential for the occurrence of pseudocritical NCF lines and extra peaks of $c_{m}$ within an unfrustrated Ising model approximation.

It should be noticed, however, that all of the Ising-type model calculations ${ }^{1-3}$ neglect the onset of transverse fieldinduced spin precession in the antiparallel sublattice for $H \rightarrow H_{c}$ as evidenced by Mössbauer spectroscopy. ${ }^{4}$ A full account of the spin dynamics should, hence, include a finite value of the single ion anisotropy ${ }^{5}$ within an anisotropic Heisenberg model. Interestingly, various important aspects of the PD of $\mathrm{FeBr}_{2}$ were recently ${ }^{6}$ found within the framework of a Hubbard model, which is usually thought to be appropriate for itinerant magnetic systems. According to these quantum Monte Carlo calculations the MCP is decoupled into a critical endpoint (CE) at $T_{m}$ and a bicritical endpoint (BCE) at $T \approx 1.3 T_{m}$ connected by a first-order PT line. It separates two AF phases with low and high axial magnetization, $\mathrm{AF}_{1}$ and $\mathrm{AF}_{2}$, respectively. Similar decoupling of the TCP also occurs in anisotropic Heisenberg AF model systems with weak next-nearest FM exchange and intermediate single ion anisotropy. ${ }^{7}$ In fact, recent specific heat data taken on $\mathrm{FeBr}_{2}$ in axial magnetic fields ${ }^{3}$ reveal sharp anomalies at $H_{-}(T)$. They strongly favor the predicted ${ }^{6,7} \mathrm{AF}_{1}-\mathrm{AF}_{2}$ PT scenario, although magnetization measurements ${ }^{1}$ merely show weak anomalies at $H_{-}(T)$.

Clearly both the $c_{m}$ data ${ }^{3}$ and the conjectured $\mathrm{PD}^{6,7}$ call for detailed investigations. In this article we present novel ac susceptibility, light diffraction, and Faraday contrast microscopy data, which partly confirm the above scenario. In particular, metamagnetic domains appearing both below and above $T_{m}$, seem to corroborate the PT signature of the NCF line $H_{-}(T)$. The experiments were carried out on thin platelets (thickness $t \approx 0.2 \mathrm{~mm}$ ) cleaved parallel to the $c$ plane of Bridgman-grown single crystals. The optical experiments involved a He gas flow cryostat equipped with a superconducting solenoid, ${ }^{1}$ a laser diode at $\lambda=670 \mathrm{~nm}$, a photomultiplier, and a charge-coupled device (CCD) camera. The ac susceptibility measurements were performed at a frequency of $f=1$ $\mathrm{Hz}$ with a SQUID magnetometer (Quantum Design MPMS $5 \mathrm{~S}$ ) in magnetic fields up to $H=3.5 \mathrm{MA} / \mathrm{m}$ and temperatures $3.5<T<10 \mathrm{~K}$.

Figure 2(a) shows the formation of AF and PM stripe domains with individual widths $w \approx(5 \pm 1) \mu \mathrm{m}$ visualized by Faraday microscopy at $T=3 \mathrm{~K}$ and $H=2.5 \mathrm{MA} / \mathrm{m}$. It is noticed that the Faraday contrast decreases when approaching the horizontal mixed phase boundaries, $H_{-}(T)$ and $H_{+}(T)$. Surprisingly, it persists up to $T=5.5 \mathrm{~K}$ when crossing the vertical boundary at $T_{m}$ under constant intermediate field $H \approx\langle H\rangle=\left(H_{c 1}+H_{c 2}\right) / 2$ [Fig. 2(b) and Fig. 1, hatched area]. This seems to corroborate the predicted ${ }^{6,7}$ first-order PT line between $\mathrm{CE}(=\mathrm{MCP})$ and $\mathrm{BCE}$, which splits into a demagnetization-induced mixed phase with coexisting $\mathrm{AF}_{1}-\mathrm{AF}_{2}$ stripe domains.

The observed domain patterns with quasi-periodic variations of the circular refractive index give rise to anisotropic diffraction of light. Figure 3 shows intensity profiles of diffraction patterns obtained with linearly polarized light passing the magnetic grating and an analyzer oriented either par- 


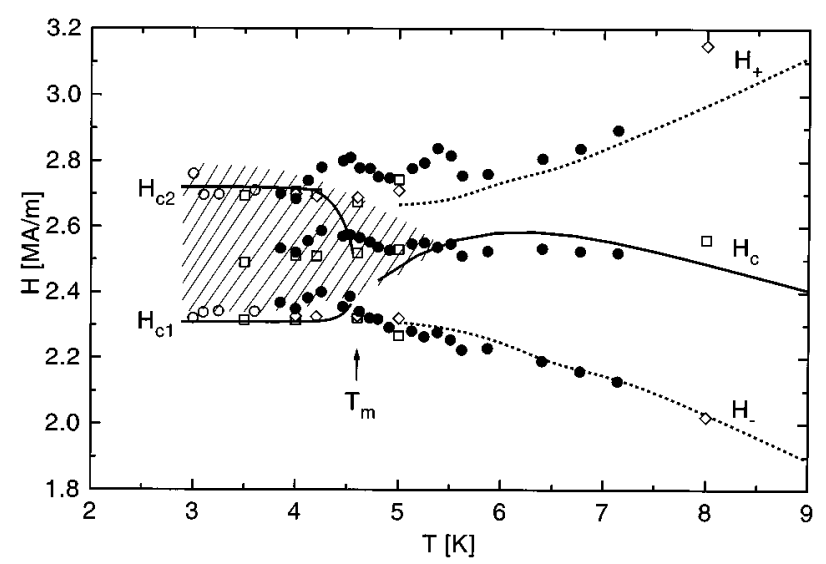

FIG. 1. Magnetic phase diagram of $\mathrm{FeBr}_{2}$ (solid and broken lines; see text) determined by magnetization measurements (see Ref. 1) in the vicinity of $T_{m}=4.6 \mathrm{~K}$ (arrow). Marginal and peak positions of light diffraction efficiency (open and solid circles, respectively) and $\chi^{\prime}(H)$ (open squares), peak positions of $\chi^{\prime \prime}(H)$ (open diamonds) and the range of domain observation (hatched area) are indicated.

allel (curves 1 and 2 at $H=0$ and $2.5 \mathrm{MA} / \mathrm{m}$, respectively) or perpendicularly to the polarizer (curve $3, H=2.5 \mathrm{MA} / \mathrm{m}$ ). It demonstrates

(i) the field-induced onset of diffraction,

(ii) the mutually orthogonal polarizations of zeroth and first-order diffracted light as expected from scalar magnetic diffraction theory, where the weak depolarization of the zeroth order is due to slight differences of the AF and PM stripe widths, and

(iii) the average grating period $\Lambda=(\lambda z) /(\langle x\rangle\langle n\rangle) \approx(10$ $\pm 2) \mu \mathrm{m}$ being in good agreement with the observed stripe width, $w=\Lambda / 2$, where $\langle x\rangle \approx(12 \pm 2) \mathrm{mm}$ is the center of gravity of the first-order peak position, $z=305 \mathrm{~mm}$ its distance from the crystal, and $\langle n\rangle \approx 1.7$ the average axial refractive index.

In order to detect mixed phases with regular or random magnetic domain structures it is convenient to register the decrease of the zeroth-order intensity, $I_{0}$, passing an appropriate diaphragm. ${ }^{8}$ Figure 4 shows the field dependence of $I_{0}$ for circularly polarized laser light at $\lambda=670 \mathrm{~nm}$. At $T$ $\leqslant 3.6 \mathrm{~K}$ the metamagnetic domain structure (see above) gives rise to a conventional ${ }^{8}$ minimum sharply setting in at the mixed phase boundaries (arrows in Fig. 4; open circles in

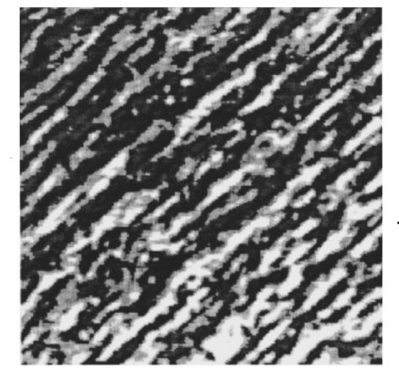

(a)

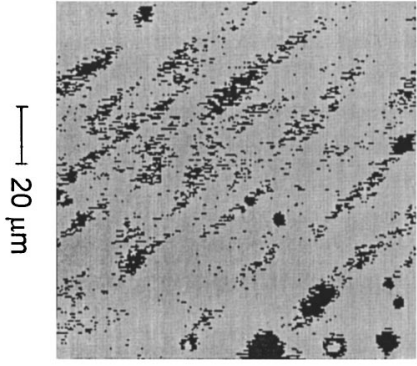

(b)
FIG. 2. Faraday contrast patterns of stripe domains in a $\mathrm{FeBr}_{2}$ sample obtained at $H=2.5 \mathrm{MA} / \mathrm{m}$ at (a) $T=3.0$ and (b) $4.9 \mathrm{~K}$, respectively.

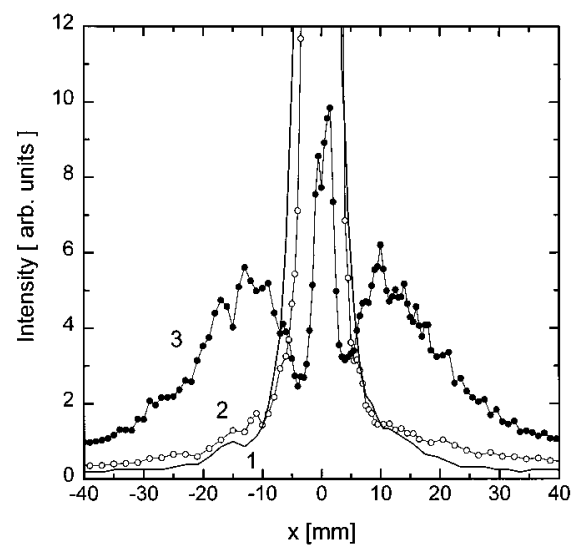

FIG. 3. Diffracted intensity vs detector position $x$ for linearly polarized 670 nm light passing an $\mathrm{FeBr}_{2}$ sample along its $c$ axis and an analyzer oriented parallel (curves 1 and 2 at $H=0$ and $2.5 \mathrm{MA} / \mathrm{m}$, respectively) and perpendicularly to the polarizer, respectively (curve $3, H=2.5 \mathrm{MA} / \mathrm{m}$ ).

Fig. 1) and peaking at $\langle H\rangle$, where $\mathrm{AF}$ and $\mathrm{PM}$ volume fractions are equipartitioned. Surprisingly, above $T \approx 3.7 \mathrm{~K}$ the single minimum of $I_{0}(H)$ transforms into three minima, which remain visible up to fairly high temperatures before becoming unstable above $T \approx 7.2 \mathrm{~K}$ (arrows in Fig. 4; solid circles in Fig. 1). Since previous experiments ${ }^{9}$ did not reveal such a fine structure within the mixed phase of $\mathrm{FeBr}_{2}$, we cannot rule out a sample dependent field-induced modulation of the Faraday contrast. On the other hand, the close resemblance of the triplet structures of $I_{0}(H)$ found below and above $T_{m}$ suggests a peculiar, albeit unexplained continuation of the spatial inhomogeneities between the mixed phase regions, AF-PM and $\mathrm{AF}_{1}-\mathrm{AF}_{2}$. Further experiments are necessary to clarify this phenomenon.

Above $T_{m}$ the transmission minima coincide within er-

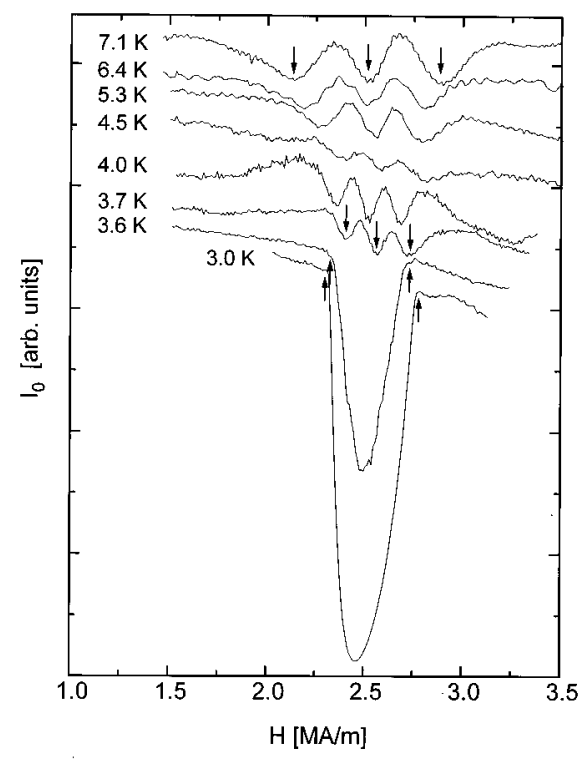

FIG. 4. Zeroth-order intensity $I_{0}$ vs applied field $H$ for circularly polarized $670 \mathrm{~nm}$ light passing an $\mathrm{FeBr}_{2}$ sample along its $c$ axis at various temperatures as indicated. Margins and minima for the arbitrarily shifted curves are marked by arrows. 


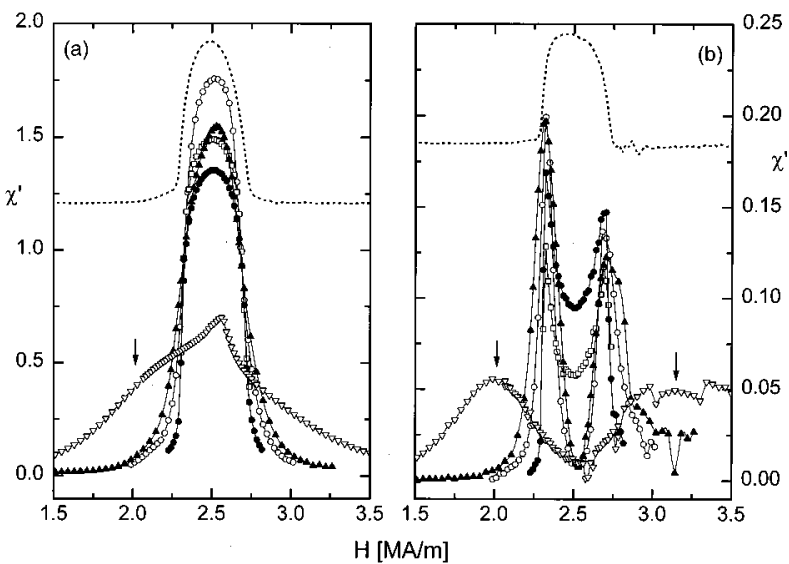

FIG. 5. (a) $\chi^{\prime}(H)$ and (b) $\chi^{\prime \prime}(H)$ measured at $T=3.5 \mathrm{~K}$ and $f=20 \mathrm{~Hz}$ (see Ref. 1) (dotted lines; arbitrarily shifted) and at $T=4.0$ (solid circles), 4.2 (open squares), 4.6 (open circles), 5.0 (solid triangles) and $8.0 \mathrm{~K}$ (open triangles), respectively. Shoulder (a) and peak (b) positions are indicated by arrows.

rors with the lines $H_{c}, H_{-}$and $H_{+}$vs $T$. Since light diffraction responds to both static and dynamic domain structures, its correlation with slow NCF at $H_{-}$and $H_{+}$is not surprising. Obviously their correlation length is of the order of $\lambda$ and thus close to that of the static domains seen in nearby $H$ - $T$ regions (Fig. 1). The same arguments apply to the observed scattering along the critical line, $H_{c}(T)$, being due to critical fluctuations with large coherence lengths.

Similar information is obtained from the field dependence of the complex low frequency susceptibility, $\chi^{\prime}-i \chi^{\prime \prime}$. Figure 5(a) shows the real part, $\chi^{\prime}(H)$, for $T=4$, $4.2,4.6,5$, and $8 \mathrm{~K}$ at $f=1 \mathrm{~Hz}$ and, in addition, for $T=3.5 \mathrm{~K}$ at $f=20 \mathrm{~Hz}^{1}$ (dotted line). At $T<T_{m}$ we find broad maxima with rounded peaks at $\langle H\rangle$ and steepest descents at $H_{c 1}$ and $H_{c 2}$ (Fig. 1, open squares). These signals are compatible with the density of domain boundaries expected within the mixed phase. Above $T_{m}$ the peaks sharpen and closely follow the PT line, $H_{c}(T)$, whereas the lateral points of inflexion coincide with the NCF lines up to $T \approx 7 \mathrm{~K}$ (Fig. 1, open squares). At higher temperatures a shoulder appears at $H_{-}$ [Fig. 5(a) arrow]. More clearly, this is confirmed by welldefined peaks of the imaginary part, $\chi^{\prime \prime}(H)$ [Fig. 5(b) ar- rows], which closely follow the lines $H_{-}, H_{+}, H_{c 1}$ and $H_{c 2}$ vs $T$, respectively (Fig. 1, open diamonds).

Upon cooling to below $T_{m}$ the weak $\chi^{\prime \prime}$ signal between $H_{c 1}$ and $H_{c 2}$ gradually increases until becoming a rounded peak at $T=3.5 \mathrm{~K}$. This seems to indicate a decrease of the domain wall losses when approaching $T_{m}$ from below, which might be due to critical speeding-up of the domain wall dynamics in the vicinity of the MCP (or CE). Such a behavior is well-known from ferromagnetic domain wall dynamics near to $T_{c} \cdot{ }^{10}$ It will be interesting to investigate the frequency dispersion of $\chi^{\prime \prime}$ in more detail. It seems to be controlled by extremely slow relaxation both in the domain wall regime below and in the NCF regime above $T_{m}$ except near to the critical region. This observation seems, again, to corroborate the conjectured continuation of the first-order PT line to above $T_{m}$.

In conclusion, our experiments seem to provide evidence for the instability of the TCP in $\mathrm{FeBr}_{2}$ as predicted by model calculations. ${ }^{2,6,7}$ The appearance of static stripe domains both below and above $T_{m}$, the occurrence of light diffraction at both static domains and slowly relaxing NCF, and the virtual continuation of the lower first-order PT line from $H_{c 1}(T)$ to $H_{-}(T)$ are in agreement with a first-order PT line intercepted by a CE at $T_{m}$ and ending with a BCE, tentatively above $T \approx 7.2 \mathrm{~K}$. Very probably, ${ }^{1,3}$ the upper NCF line, $H_{+}(T)$, is fluctuation-induced and does not define an equilibrium phase boundary.

This work was supported by the Deutsche Forschungsgemeinschaft.

${ }^{1}$ M. M. P. de Azevedo, Ch. Binek, J. Kushauer, W. Kleemann, and D. Bertrand, J. Magn. Magn. Mater. 140-144, 1557 (1995).

${ }^{2}$ W. Selke and S. Dasgupta, J. Magn. Magn. Mater. 147, L245 (1995); W. Selke, Z. Phys. B 101, 145 (1996).

${ }^{3}$ H. Aruga Katori, K. Katsumata, and M. Katori, Phys. Rev. B 54, R9620 (1996).

${ }^{4}$ J. Pelloth, R. A. Brand, S. Takele, M. M. Pereira de Azevedo, W. Kleemann, Ch. Binek, and J. Kushauer, Phys. Rev. B 52, 15372 (1995).

${ }^{5}$ L. Hernandez, H. T. Diep, and D. Bertrand, Europhys. Lett. 21, 711 (1993).

${ }^{6}$ K. Held, M. Ulmke, and D. Vollhardt, Mod. Phys. Lett. B 10, 203 (1996).

${ }^{7}$ I. Vilfan and S. Galam, Phys. Rev. B 30, 6428 (1986).

${ }^{8}$ C. Binek and W. Kleemann, J. Phys., Condens. Matter. 5, 3457 (1993).

${ }^{9}$ T. E. Wood, A. Muirhead, and P. Day, J. Phys. C 11, 1619 (1978).

${ }^{10}$ M. Grahl and J. Kötzler, Z. Phys. B 75, 527 (1989). 